\title{
SIOP/COG/NWTSG y-Stage I
}

National Cancer Institute

\section{Source}

National Cancer Institute. SIOP/COG/NWTSGy-Stage I. NCI Thesaurus. Code C140302.

The assessment of stage I Wilms' tumor was performed after neoadjuvant therapy was given. 